\title{
A Pilot Study of a 6-Week Parenting Program for Mothers of Pre-school Children Attending Family Health Centers in Karachi, Pakistan
}

\author{
Yasmin Khowaja $^{1^{*}}$, Rozina Karmaliani ${ }^{1,2}$, Shela Hirani ${ }^{1}$, Asif Raza Khowaja ${ }^{3}$, Ghazala Rafique ${ }^{2}$, Judith McFarlane ${ }^{4}$
}

\begin{abstract}
Background: Recently, parenting programs to address behavioural and emotional problems associated with child maltreatment in developing countries have received much attention. There is a paucity of literature on effective parent education interventions in the local context of Pakistan. This study aimed to assess the feasibility of offering a 6-week parenting program for mothers of pre-school children attending family health centres (FHCs) in Karachi, the largest metropolitan city of Pakistan.

Methods: A pilot quasi-experimental trial was conducted. Two FHCs were selected, one as the intervention and the second as the control. A total of 57 mothers of pre-school children $(n=30$ intervention; $n=27$ control) participated in this study. Mothers in the intervention group received SOS Help for parents module, while mothers in the control group received information about routine childcare. A parenting scale (PS) was administered before the program was implemented and repeated 2 weeks after the program was completed in both groups. Statistical analysis was performed to compare participants' attributes. Descriptive analysis was conducted to compare pre- and post-test mean scores along with standard deviation for parenting subscales in the intervention and control groups.

Results: A total of 50 mothers ( $n=25$ intervention; $n=25$ control) completed the 6 -week program. Attrition was observed as $5 / 30(17 \%)$ in the intervention arm and $2 / 27(2 \%)$ in the control arm. Mothers commonly reported the burden of daily domestic and social responsibilities as the main reason for dropping out. Furthermore, the majority of participants in the control group recommended increasing the duration of weekly sessions from 1 to 1.5 hours, thereby decreasing the program period from 6 to 4 weeks. Mothers in intervention group reported substantial improvement in parenting skills as indicated by mean difference in their pre- and post-test scores for laxness and over-reactivity.

Conclusion: Parenting programs can be implemented for mothers attending FHCs in Pakistan. Mothers require positive reinforcement and constant encouragement at the participant level. Integrating such programs into primary healthcare at the population level has the potential to maximize child health benefits and to improve parenting skills at the country level.

Keywords: Parenting Program, Child Maltreatment, Child Abuse, Education for Mothers

Copyright: (C) 2016 by Kerman University of Medical Sciences

Citation: Khowaja Y, Karmaliani R, Hirani S, Khowaja AR, Rafique G, McFarlane J. A pilot study of a 6-week parenting program for mothers of pre-school children attending family health centers in Karachi, Pakistan. Int J Health Policy Manag. 2016;5(2):91-97. doi:10.15171/ijhpm.2015.181
\end{abstract}

\section{Article History:}

Received: 9 March 2015

Accepted: 1 October 2015

ePublished: 11 October 2015

*Correspondence to:

Yasmin Khowaja

Email: ysak6@hotmail.com

\section{Key Messages}

Implications for policy makers

This study suggested implications for primary health practice, research, and policy for integrating parenting programs into health service delivery in Pakistan.

- Sensitizing healthcare providers to the importance of parenting education could ultimately lead to better-designed maternal and child health promotion programs at the community level. The benefits of parenting classes, specifically in terms of the positive outcomes for mothers and children, need to be communicated to frontline healthcare providers working at primary health centres, such as medical doctors and paramedics.

- Parenting education could be integrated into community-based programs by involving outreach community care providers. Primary health centres could potentially bridge the service-delivery gap for acute child behavioural problems or disorders by involving community care providers. For example, Lady Health Workers of the National Program are already providing basic preventive maternal and child health services, and could potentially be engaged in parenting education programs.

- Further research is needed to understand the implications of policy to streamline parenting programs as part of primary healthcare delivery. Very limited knowledge is currently available and more is needed on issues such as system-capacity and healthcare providers' willingness to participate.

- Partnering with the media could strengthen arguments for introducing parenting education into the service-delivery model of healthcare by providing a voice to unheard people with stories of child abuse and maltreatment.

Implications for public

This study demonstrated the feasibility of offering parenting programs for mothers in 2 communities in Pakistan. Such programs provide mothers with an opportunity to learn a new set of skills, and contribute to healthy childcare practices at home and in the community. The positive parenting has the potential to improve one's overall quality of life and to reduce behavioural problems/disorders in children. Furthermore, effective parenting improves cognitive and intellectual capabilities of young children, which ultimately results in high academic and professional performance in later years of life. 


\section{Background}

Child maltreatment is a global issue that affects children physically, psychologically and developmentally throughout their lives. ${ }^{1}$ Child maltreatment can result in poor selfesteem, lack of confidence, and mental stress. ${ }^{2}$ The estimated prevalence of child maltreatment ranges from $4 \%$ to $16 \%$ for physical abuse and $10 \%$ for psychological abuse in highincome countries. ${ }^{3}$ Literature indicates a high burden of child maltreatment in low- and middle-income countries (LMICs), where practices for childrearing are rooted in punitive and coercive cultures. ${ }^{4}$ A review of data from 28 LMICs revealed that $83 \%$ of children were being psychologically abused and that $43 \%$ were severely physically abused. ${ }^{5}$ Besides sociodemographic and cultural factors, poor parenting practices are important determinants for child maltreatment. ${ }^{6,7} \mathrm{~A}$ recent qualitative study of abused children reported negative perceptions associated with poor parenting practices including aggressive or inconsistent disciplinary styles, neglectful behaviors, authoritative decision-making, and failure to set appropriate boundaries. ${ }^{8}$ Thus, children who lack adequate parental attention and support are more vulnerable to child abuse inside and outside home boundaries. ${ }^{9}$

Recently, parenting programs have received a great amount of attention to address behavioral and emotional problems associated with child maltreatment, particularly in the context of LMICs. ${ }^{4,10}$ Parenting programs are interventions designed to improve parenting roles through training, support and education. These programs focus on child behavioral problems, effective ways of parent-child communication and strategies to engage children in healthy activities. ${ }^{11}$ Randomized controlled trials conducted elsewhere found parenting interventions effective in improving parent-child interactions and in increasing parental knowledge about child developmental needs. ${ }^{12}$ Likewise, a meta-analysis of 23 studies on parenting programs suggested significant improvements in parents' attitudes towards preventing abuse, emotional adjustments, and childrearing skills. ${ }^{13}$ Most importantly, the World Health Organization's (WHO's) guidelines highly recommended parenting programs as an essential tool for improving children's physical health, and enhancing their cognitive development. ${ }^{14}$

In Pakistan, the rising number of child abuse cases often make newspaper headlines, ${ }^{15}$ and related public health concerns are highlighted on project reports published by non-governmental organizations (NGOs). ${ }^{16}$ However, the scientific literature surrounding the magnitude of child abuse is scarce due to a lack of proper reporting and recording systems. ${ }^{17}$ It is feared that failure to prevent child abuse will increase the burden of poor child health outcomes in the country. ${ }^{18}$ The primary objective of this study was to assess the feasibility of offering a 6-week parenting program for mothers of pre-school children attending family health centres (FHCs) in Karachi, Pakistan. The secondary objective was to determine the improvement in parenting skills after completing the parenting program.

\section{Methods}

A quasi-experimental pilot trial was conducted between April and July 2013. The choice of quasi-experimental design was imperative because individual level randomization was not possible in the selected setting.
Study Settings

Aga Khan Health Service is a civil society organization which has established several FHCs across the country as part of the public-private partnership initiative for primary care in Pakistan. ${ }^{19}$ These FHCs provide a range of similar health services such as vaccinations, medical consultations, antenatal-and-postnatal consultations, family planning, and health education. Through our institutional collaboration, we selected 2 distantly located FHCs in the metropolitan city of Karachi. One FHC was assigned as the intervention and the other as the control using a flip of a coin. The assignment of the FHCs was done in the presence of FHC administrative staff, the study coordinator, and the primary investigator.

\section{Inclusion and Exclusion Criteria}

Mothers were considered eligible if they met the following three criteria: the purpose of their visit to the FHC was related to routine childcare (eg, the vaccination of children aged 2-5 years), they lived in the catchment areas of the selected FHCs and they were available for the time that was to be dedicated to the program ( 6 consecutive weeks). Mothers were excluded if any of the following applied: the purpose of their visit was related to the management/treatment of chronic childhood illnesses or any medical emergencies, they lived outside of the catchment areas of the selected FHCs and they were unavailable for 6 consecutive weeks. Mothers who refused to consent to the study were also excluded (Figure 1).

\section{Parenting Sessions for Mothers in the Intervention and Control Arms}

This study included one intervention and one control arm. Mothers recruited to either arm were subsequently assigned to smaller groups according to their availability for weekly sessions. The intervention arm included 3 groups (ie, group-1 $\mathrm{n}=15$, group $-2 \mathrm{n}=3$, and group $-3 \mathrm{n}=12$ ). Group -2 comprised of 3 working mothers, therefore, sessions were arranged in the afternoon. The control arm included 2 groups (ie, group-1 $n=19$, group- $2 n=8$ ). A 6-week session schedule was distributed to all participants in advance. The participants were allowed to join parallel sessions scheduled for other small groups at the same FHC if they were unable to attend any particular session. Reminders were generated using phone calls to all participants one day before each session. A recruitment log, which included the mother's name, age of her youngest child, residential address, phone number and preferred day/time for the sessions, was maintained at each site. The same registered nurse, who was also involved in the Urdu translation of the module, conducted all the sessions in the intervention and control arms. Participatory learning strategies included group discussion, self-reflection, roleplaying demonstrations, and watching videos, were employed to effectively deliver the weekly program content. The content and its implementation were supervised by 2 independent researchers with academic backgrounds in both nursing and early childhood development (ECD). Weekly attrition and strategies for participant retention were discussed in biweekly meetings with the investigators alongside other matters.

\section{Intervention}

The intervention was a demonstration of positive parenting 


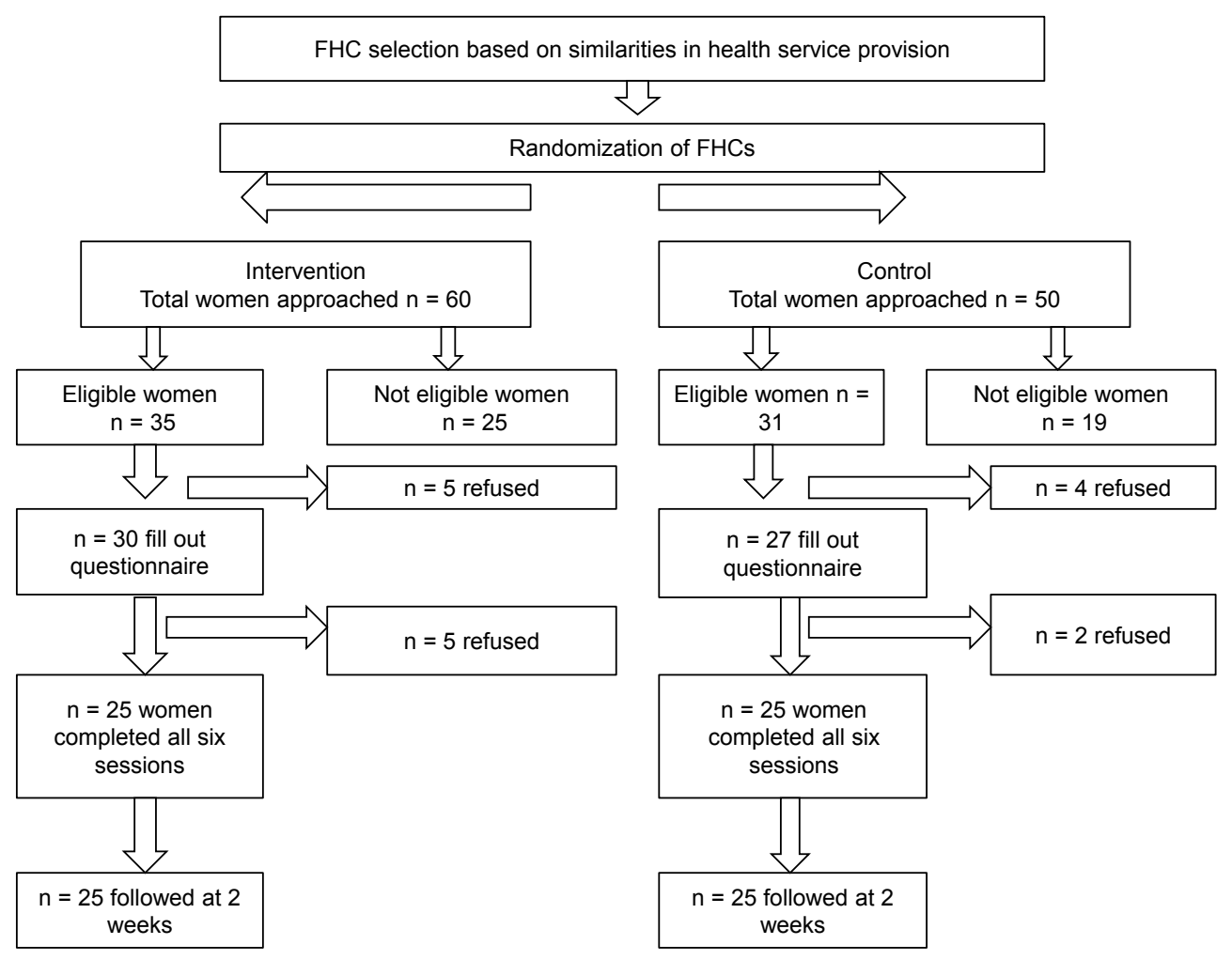

Figure 1. Participant Recruitment in Intervention and Control Arms. Abbreviation: FHC, family health centre.

skills using the SOS Help for Parents module. ${ }^{20}$ We chose this module because it has been highly recommended for healthcare professionals such as psychologists, pediatricians, and nurses, as well as for parents. Also, previous intervention studies from Iran and Iceland reported significant improvements in parenting skills after using the same module. ${ }^{21,22}$ This module has been translated into 9 different languages and widely used in both developed and developing countries. As the module had not yet been translated into the national language of Pakistan, Urdu, at the time of the study, an English version was requested from the SOS Programs and Parents Press Company and, with permission, translated into Urdu.

The SOS Help for parents module focuses on 46 different child behavioral problems and 20 basic child management strategies. The program covered 6 major themes, presented and discussed during 6 weekly, 1-hour sessions. The Urdutranslated brochures on child management methods and 'time-out' strategies were distributed to all the participants during the first session. Every session thereafter, participants watched program videos and were asked to self-reflect on the past week's activities. Week one introduced the parenting guidebook as a whole and delved into topics such as the explanation of misbehavior in children. Week 2 began discussions related to childrearing, and week 3 continued this topic, focusing on the top 4 errors to avoid. Week 4 focused on the importance of effective communication; week 5 on determining ways to encourage good behavior from children; and week 6 on teaching parents how to respond to bad behavior from children, such as various effective punitive measures. Also in this final week, participants provided feedback regarding the project's strengths, areas of improvement, and future recommendations.
Control

Mothers in the control group received health education for routine childrearing and caring practices covered in weekly, 1-hour sessions for a total of 6 consecutive weeks. The health education topics included 6 diverse topics. First and second weeks were devoted to promoting healthy lifestyles for both mothers and children: first week focused on healthy eating practices, while second week was geared towards how to keep children engaged in physical activity and/or sports after school hours. The third week focused on the specific theme of communication strategies for grandparents involved in childcare at home. The next week delved into the topic of normal growth and development milestones of children between the ages of 2 and 5 years. The fifth week dealt with similar issues, but was focused on dispelling myths about the growth and development of toddlers. The sixth week was simply a reflection on the previous 5 weeks. Evaluations of the program were also taken from all the participants during this last week.

\section{Sample Size}

Previous studies found parenting programs to be very effective, and the effectiveness estimates ranged between 30\% to $50 \%$ depending on the type of project and its length..$^{23,24}$ Keeping in mind feasibility as the primary objective of our study, we required a fairly small sample. We calculated sample size using $80 \%$ power, $95 \% 2$-sided level of significance, 1 as the ratio of sample size exposed/unexposed and medium effect size of $40 \%$. The estimated sample size was 50 ( 25 for the intervention group and 25 for the control group). Using $10 \%$ refusal and $10 \%$ attrition rate, the final sample size was inflated to a total of 60 mothers (ie, 30 in each group). 


\section{Methods of Data Collection}

Consistent methods were used to collect data from participants in both the intervention and control arms. A socio-demographic questionnaire was administered and key variables included ages of mothers and children, number of children, years of schooling, occupations, and family structures. We used the parenting scale (PS) to evaluate the effectiveness of parenting interventions as it has been utilized by similar studies elsewhere. The PS is a 30 -item, selfreported scale that broadly categorizes parenting strategies into 3 factors/subscales: laxness (permissive discipline), over-reactivity (authoritarian discipline, displays of anger, meanness, and irritability), and hostility/verbosity (overly long reprimands or reliance on talking). ${ }^{25} \mathrm{~A}$ previous study reported validity measures for PS factors as coefficient alpha of $0.83,0.82,0.63,0.84$ for laxness, over-reactivity, hostility/verbosity, and total factor, respectively. The testretest correlations were reported as 0.83 for laxness, 0.82 for over-reactivity, 0.79 for hostility/verbosity and 0.84 as the total. ${ }^{26}$ We restricted our analysis on 2 factor solutions - laxness and over-reacitvity - based on the reported high validity and reliability measures. The PS was distributed to mothers in the intervention and control arms as a pre-test before implementing the parenting program; it was repeated as a post-test 2 weeks after the completion of the program. The nurse maintained a reflective diary for all the sessions, in which she recorded personal experiences, length of the sessions, operational difficulties, and logistical problems. This greatly helped in addressing operational challenges during the implementation of the program. Participants' attendance and reasons for attrition were documented after each weekly session.

\section{Data Quality}

The parenting module and PS were translated from English to Urdu and back translated by 2 language experts in order to maintain content accuracy. Prior to the start of the intervention, the module content and PS questionnaires were tested on $10 \%$ of the total sample size in a similar setting. This helped simplify language translation, comprehension of the content by participants and estimate duration for each session. The double entry of data was done on the program Visual FoxPro (version 7) by 2 independent persons. Prior to data analysis, data cleaning and consistency checks were established to further minimize chances of error.

\section{Data Analysis}

Chi-square and $t$ test statistics were calculated to compare participants' attributes. Descriptive analysis was performed to plot weekly participation, and pre- and post-test mean scores along with standard deviation (SD) for parenting subscales in the intervention and control groups. Data analysis was performed on SPSS version 19.

Results

A total of 57 mothers (30 in the intervention group and 27 in the control group) were recruited to this study. Participants in both groups were similar in terms of ages, number of children in the family, children's ages, and number of people living in the households. However, there was a significant difference observed in the mean household income for mothers in the intervention and control arms (Table 1).

Other variables such as native languages, marital status, family structures, educational level of mothers, mothers' occupations, and husbands' occupations were not significantly different in either group (Table 2).

\section{Participants' Attrition at Weekly Sessions}

Attrition varied between the intervention and control groups during the first 3 weeks and started to stabilized in subsequent weeks. In the first week following recruitment, attrition was higher $(\mathrm{n}=3,10 \%)$ in the intervention arm as compared to the control arm $(\mathrm{n}=1,4 \%)$. In the second week, attrition occurred only in the intervention arm $(n=1,4 \%)$. Participant retention improved after the third week, and only 1 participant refused during the fifth week in the intervention group. Overall, attrition was observed at 5/30 (17\%) in the intervention group, and $2 / 27(2 \%)$ in the control group (Figure 2).

\section{Participant Feedback and Reasons for Attrition on Weekly Sessions}

The majority of participants in both the intervention and control groups considered the 6-week program period too long. They recommended increasing the duration from 1 to 1.5 hours per session, thereby decreasing the weekly program period from 6 to 4 weeks. Participants appreciated the program content in the intervention arm, and both groups liked the participatory learning strategies. A few participants in the intervention arm also recommended to contextually customize the program content to include grandparents' roles and responsibilities in childcare.

In response to attrition, mothers in both arms reported similar concerns related to time constraints and the burden of household chores as main barriers for not completing the 6-week program (Table 3).

Comparison of Pre- and Post-test Mean Scores for Parenting Subscales

Mothers in the intervention group reported substantial improvement in their parenting skills as indicated by the mean difference in pre- and post-test scores for laxness and

Table 1. Participants' Attributes in Intervention and Control Arms

\begin{tabular}{|c|c|c|c|c|}
\hline Variables & $\begin{array}{c}\text { Control }(n=27) \\
\text { Mean (SD) }\end{array}$ & $\begin{array}{c}\text { Intervention }(n=30) \\
\text { Mean }(S D)\end{array}$ & $\mathbf{t}$ & $P$ Value \\
\hline Mother's age (years) & $31.56(5.33)$ & $29.67(3.87)$ & 1.540 & 0.129 \\
\hline Number of children in family & $1.85(0.77)$ & $1.90(0.80)$ & -0.230 & 0.819 \\
\hline Child's age (years) & $3.36(1.10)$ & $3.20(1.03)$ & 0.736 & 0.465 \\
\hline Number of people living in the family & $4.78(1.25)$ & $4.93(1.76)$ & -0.381 & 0.705 \\
\hline Monthly household income in thousands (PKR) & $30.00(16.74)$ & $20.03(9.50)$ & 2.819 & 0.007 \\
\hline
\end{tabular}


Table 2. Participants' Attributes in Intervention and Control Arms

\begin{tabular}{|c|c|c|c|c|}
\hline Variables & $\begin{array}{c}\text { Control }(n=27) \\
\text { No. }(\%)\end{array}$ & $\begin{array}{c}\text { Intervention }(\mathrm{n}=30) \\
\text { No. }(\%)\end{array}$ & $x^{2}$ & P Value \\
\hline Mother's native language & & & 5.146 & 0.273 \\
\hline Urdu & $15(55.60)$ & $8(26.66)$ & & \\
\hline Sindhi & $5(18.52)$ & $8(26.66)$ & & \\
\hline Gujarati & $2(7.41)$ & $3(10.00)$ & & \\
\hline Punjabi & $2(7.41)$ & $4(13.33)$ & & \\
\hline Others & $3(11.06)$ & $7(23.35)$ & & \\
\hline Marital status & & & 2.011 & 0.366 \\
\hline Married & $26(96.29)$ & $29(96.67)$ & & \\
\hline Divorced & $1(3.71)$ & $0(0.00)$ & & \\
\hline Widowed & $0(0.00)$ & $1(3.33)$ & & \\
\hline Family structure & & & 0.740 & 0.390 \\
\hline Nuclear & $15(55.55)$ & $20(66.67)$ & & \\
\hline Extended & $12(44.45)$ & $10(33.33)$ & & \\
\hline Mother's education level & & & 1.067 & 0.785 \\
\hline Illiterate & $0(0.00)$ & $1(3.33)$ & & \\
\hline Primary/secondary & $8(29.63)$ & $10(33.33)$ & & \\
\hline College & $10(37.04)$ & $10(33.33)$ & & \\
\hline University & 9 (33.33) & $9(30.00)$ & & \\
\hline Mother's profession/occupation & & & 0.306 & 0.580 \\
\hline Housewife & $23(85.16)$ & $27(90.00)$ & & \\
\hline Working mothers & $4(14.84)$ & $3(10.00)$ & & \\
\hline Husband's profession/occupation & & & 0.848 & 0.654 \\
\hline Private job & $15(55.55)$ & $19(63.33)$ & & \\
\hline Personal business & $11(40.74)$ & $9(30.00)$ & & \\
\hline Others & $1(3.71)$ & $2(6.67)$ & & \\
\hline
\end{tabular}

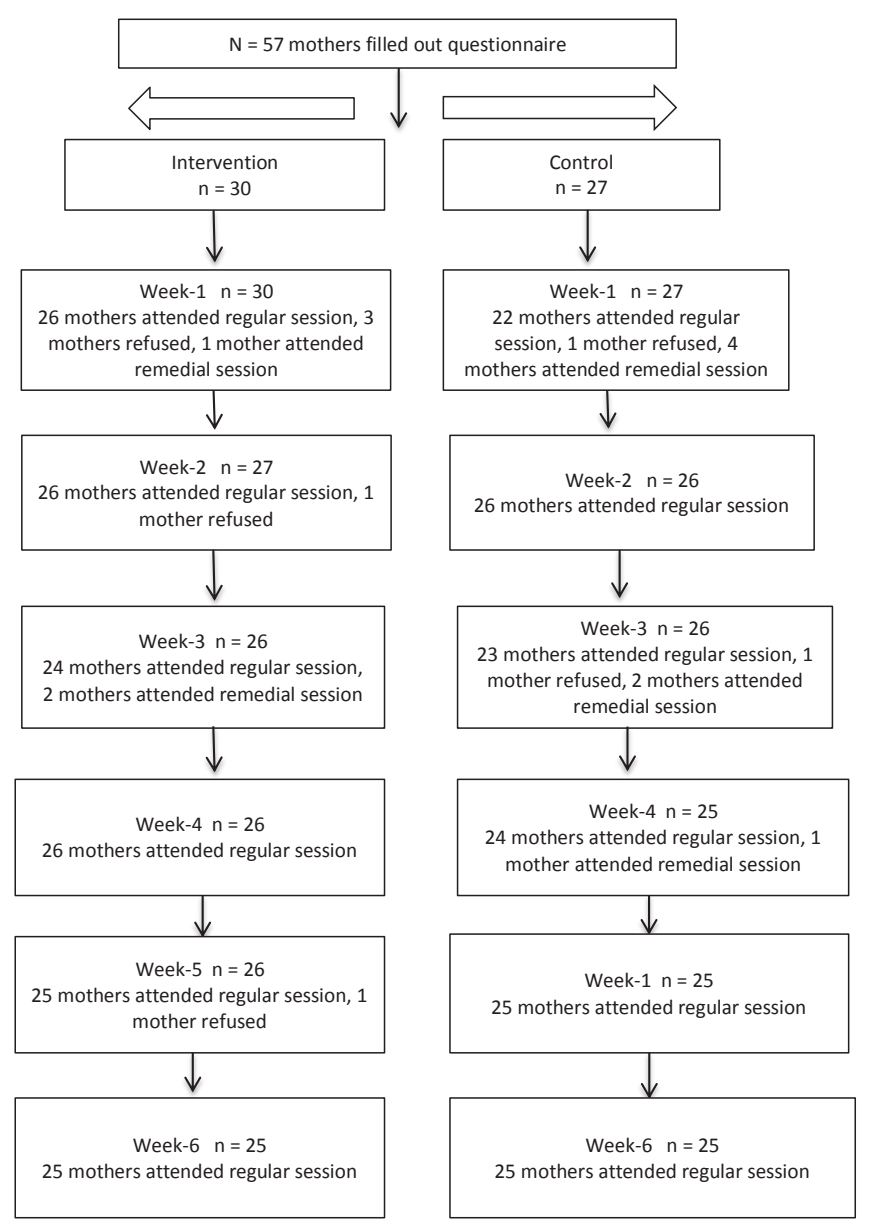

Figure 2. Recruitment and Weekly Participations in Intervention and Control Arms. over-reactivity. However, scores for mothers in the control group indicated very little improvement for laxness and overreactivity (Table 4 ).

\section{Discussion}

Prevention of child maltreatment requires a multisectoral approach. ${ }^{27}$ The WHO provided a framework of interventions for preventing child maltreatment, which is very relevant in the context of LMICs. According to that framework, the societal/community level interventions relate to implementing legal reform and human rights, introducing beneficial social and economic policies, changing cultural and social norms, and reducing economic inequalities/environmental risk factors. The individual level interventions relate to reducing unintended pregnancies, increasing access to prenatal/ postnatal services, and training for effective parenting skills. ${ }^{28}$ In line with WHO's framework for individual level interventions, our research focused on the feasibility of a 6-week parenting program for mothers in Pakistan. Program completion was higher $(n=50$ out of $57 ; 88 \%)$ in our study, compared to $82.3 \%$ and $74.5 \%$ reported in similar studies in Iran and Australia, respectively. ${ }^{21,29}$ The study conducted in Iran administered module content in weekly, 2-hour sessions over 2 successive weeks. ${ }^{21}$ The shorter session duration in our study was sensible to the needs of working mothers. Furthermore, the higher rate of participation in our study may be attributed to the recruitment and retention strategies that the researchers used to engage the participants throughout the weekly sessions.

There is growing scholarly interest in the area of serviceintegration, whereby parenting programs can be introduced 
Table 3. Reasons for Attrition on Weekly Sessions

\begin{tabular}{lcl}
\hline Groups & Reasons for attrition as reported by mothers \\
\hline \multirow{3}{*}{ Intervention } & - & Busy with moving to new residence \\
& - & Illness of family member (in-laws) \\
& - & Working late hours at job \\
Control & - & Social gatherings and meeting friends \\
& - & Illness of family member (child and in-laws) \\
\hline
\end{tabular}

Table 4. Comparison of Pre-test Mean Scores for Parenting Subscales

\begin{tabular}{lccc}
\hline Parenting Subscales & Pre-test & Post-test & $\begin{array}{c}\text { Mean } \\
\text { Difference }\end{array}$ \\
\hline Intervention group & & & \\
Mean (SD) for laxness & $4.58(0.75)$ & $1.74(0.29)$ & 2.84 \\
Mean (SD) for over-reactivity & $2.99(0.84)$ & $1.23(0.22)$ & 1.76 \\
Control group & & & \\
Mean (SD) for laxness & $4.52(0.39)$ & $4.48(0.74)$ & 0.04 \\
Mean (SD) for over-reactivity & $2.85(0.92)$ & $2.77(0.75)$ & 0.08 \\
\hline
\end{tabular}

into primary healthcare delivery. A qualitative study explored perceptions of healthcare providers and parents about parenting programs conducted at primary health centres in the United States. This study reported higher program acceptability, and perceptions of public benefit. ${ }^{30}$ In addition, a randomized controlled trial conducted in low-income minority mothers in the United States reported an increased utilization of preventive pediatric healthcare services in the groups that received parenting interventions, as compared to standard social services. ${ }^{31}$ In our study, we learned that recruiting mothers at FHCs was feasible, as many of them were already attending health centres for routine childcare and vaccinations. A similar approach was reported by studies in Iran and Japan, whereby researchers found recruitment of mothers at PHCs both feasible and sustainable for parenting programs. ${ }^{21,32}$

Our study suggested improvement in parenting skills for mothers in the intervention group, however, future research with larger samples is imperative to draw statistically valid conclusions. The preliminary findings for improved parenting as observed in our study are corroborated with other similar studies conducted in Iceland and Iran, where the same SOS Help for Parents module was used as an intervention. ${ }^{21,33}$ Studies that used a different module named the Positive Parenting Program (Triple P) also reported significant improvements in the parenting skills after the program. ${ }^{27,32}$ Other important findings from our study indicated the need for effective parenting programs that focus on grandparents' roles and responsibilities in childcare. Although we could not find relevant studies in the context of LMICs, a fairly recent study from Australia/New Zealand utilized Triple P interventions on grandparents. That study found significant reductions in child behavioral problems and improved childparent relationships. ${ }^{34}$ Such parenting programs that focus on parents, as well as grandparents, would be very relevant to meet the context-specific needs in LMICs.

\section{Strengths and Limitations}

To our knowledge, this is the first study of its kind to address the feasibility of implementing parenting programs in primary health settings in Pakistan. Quasi-experimental design was the main study limitation. Randomization at the participant level was impractical in our setting, as mothers in the community maintained social groups and indulged in frequent interactions. Response bias could be another study limitation, as the findings were based on the mothers' selfreports only. Authors recognized that the outcome measures to include child behavior was an important aspect, however, it was beyond the scope of the PS used in the current study. Other study limitations included a small sample size, time constraints to include fathers and lack of qualitative methodologies for in-depth knowledge of participants' experiences for program satisfaction.

\section{Conclusion}

This study indicated that parenting programs are feasible to implement for mothers of pre-school children attending FHCs in Pakistan. Mothers in the intervention group reported substantial improvements in their parenting skills. Future research containing both a larger sample size and inclusivity of fathers and mothers, will supplement the scientific evidence for the effectiveness of parenting interventions. Data triangulation through qualitative methods will further contribute to the knowledge of program acceptability and satisfaction.

\section{Acknowledgements}

The School of Nursing and Midwifery of Aga Khan University located in Karachi, Pakistan, funded this research as part of the Masters in Nursing Thesis program. The authors would like to acknowledge the efforts of the management and FHCs staff of Aga Khan Health Services, (AKHS) Pakistan for their logistical support and access to data collection sites. We would like to thank all the participants for their time and commitment to this study. The authors acknowledge Elizabeth Wilcox, Raziya Merani, and Zainah Merani for editorial review of this manuscript.

\begin{abstract}
Ethical issues
The FHC administration was contacted and the research protocol was discussed. A formal approval was obtained prior to randomization of FHCs into both the intervention and control arms. Participation in this study was voluntary and all eligible mothers were invited to participate. A trained member of the project staff administered a written and informed consent form and explained study details to the eligible mothers. Participant consent was obtained during the recruitment. This study received ethics approval from Aga Khan University's Ethics Review Committee (ERC 2443-SON-ERC-12). Formal permissions were obtained from administrative primary authors of the SOS Program and the PS, prior to their use in the study.
\end{abstract}

\section{Competing interests}

The authors declare that they have no competing interests.

\section{Authors' contributions}

YK conducted a literature search to conceptualize and develop the study protocol and wrote the manuscript. SH, RK, GR, and JM were all involved in designing the study, supervising data collection and reviewing the manuscript. ARK helped in data analysis and manuscript writing. All the authors read and approved the final manuscript.

\section{Authors' affiliations}

'School of Nursing and Midwifery, Aga Khan University, Karachi, Pakistan. ${ }^{2}$ Community Health Sciences Department, Aga Khan University, Karachi, Pakistan. ${ }^{3}$ Department of Paediatrics and Child Health, Aga Khan University, 
Karachi, Pakistan. ${ }^{4} \mathrm{Health}$ Promotion and Disease Prevention, Texas Woman's University, Houston, TX, USA

\section{References}

1. Westby CO. Child maltreatment: a global issue. Lang Speech Hear Serv Sch. 2007;38(2):140-148. doi:10.1044/01611461(2007/014)

2. Holt S, Helen B, Sadhbh W. The impact of exposure to domestic violence on children and young people: a review of the literature. Child Abuse Negl. 2008;32(8):797-810. doi:10.1016/j. chiabu.2008.02.004

3. Gilbert R, Cathy SW, Kevin B, David F, Elspeth W, Staffan J. Burden and consequences of child maltreatment in high-income countries. Lancet. 2009;373(9657):68-81. doi:10.1016/s01406736(08)61706-7

4. Mejia A, Rachel C, Matthew RS. A review of parenting programs in developing countries: opportunities and challenges for preventing emotional and behavioral difficulties in children. Clin Child Fam Psychol Rev. 2012;15(2):163-175. doi:10.1007/ s10567-012-0116-9

5. Akmatov MK. Child abuse in 28 developing and transitional countries-results from the Multiple Indicator Cluster Surveys Int $J$ Epidemiol. 2011;40(1):219-227. doi:10.1093/ije/dyq168

6. Pazdera AL, McWey LM, Mullis A, Carbonell J. Child sexual abuse and the superfluous association with negative parenting outcomes: the role of symptoms as predictors. J Marital Fam Ther. 2013;39(1):98-111. doi:10.1111/j.1752-0606.2011.00272.x

7. Rodriguez CM. Analog of parental empathy: association with physical child abuse risk and punishment intentions. Child Abuse Negl. 2013;37(8):493-499. doi:10.1016/j.chiabu.2012.10.004

8. Ahmed K, Laurie W, Stephen S. In their own words: abused children's perceptions of care provided by their birth parents and foster carers. Adoption \& Fostering. 2015;39(1):21-37. doi:10.1177/0308575914565068

9. Sousa C, Herrenkohl TI, Moylan CA, et al. Longitudinal study on the effects of child abuse and children's exposure to domestic violence, parent-child attachments, and antisocial behavior in adolescence. $J$ Interpers Violence. 2011;26(1):111-136. doi:10.1177/0886260510362883

10. Knerr W, Frances G, Lucie C. Improving positive parenting skills and reducing harsh and abusive parenting in low-and middleincome countries: a systematic review. Prev Sci. 2013;14(4):352363. doi:10.1007/s11121-012-0314-1

11. Bornstein MH. Positive parenting and positive development in children. In: Lerner RM, Jacobs F, Wertlieb D, eds. Handbook of Applied Developmental Science: Promoting Positive Child, Adolescent, and Family Development Through Research, Policies, and Programs. Thousand Oaks: Sage; 2003:187-209.

12. Olds DL, Sadler L, Kitzman H. Programs for parents of infant and toddlers: recent evidence from randomized trials. J Child Psychol Psychiatry. 2007;48:355-391. doi:10.1111/j.14697610.2006.01702.x

13. Lundahl BW, Nimer J, Parsons B. Preventing child abuse: a meta-analysis of parent training programs. Res Soc Work Pract. 2006;16:251-262. doi:10.1177/1049731505284391

14. World Health Organization (WHO). Preventing violence through the development of safe, stable and nurturing relationships between children and their parents and caregivers. Available from: http://apps.who.int/iris/handle/10665/44088. Published 2009

15. The News International. Child abuse cases increasing in Pakistan. http://www.thenews.com.pk/Todays-News-6-91603Child-abuse-cases-increasing-in-Pakistan. Published February 08, 2012.

16. Manizeh B. Har bacha mehfooz-safe, secure and protected. Annul Report 2013. http://sahil.org/wp-content/uploads/2014/06/
Annual-Report-2013.pdf.

17. Malik F. Determinants of child abuse in Pakistani families: parental acceptance-rejection and demographic variables. International Journal of Business and Social Science. 2010;1(1):67-80.

18. Ali M, Shahab S, Ushijima $H$, de-Muynck A. Street children in Pakistan: a situational analysis of social conditions and nutritional status. Soc Sci Med. 2004;59(8):1707-1717. doi:10.1016/j. socscimed.2004.01.031

19. Aga Khan Health Services Pakistan website. http://www.akdn org/akhs. Updated 2007.

20. SOS Help. Helping people help themselves. Programs and Parents Press. http://www.sosprograms.com/index.html.

21. Oveisi S, Ardabili HE, Dadds MR, et al. Primary prevention of parent-child conflict and abuse in Iranian mothers: A rondomized-controlled trial. Child Abuse Neglect. 2010;34:206213. doi:10.1016/j.chiabu.2009.05.008

22. Clark, L. SOS Help for Parents. 3rd ed. Bowling Green, USA: SOS programs and parents press; 2005.

23. Cowen PS. Effectiveness of a parent education intervention for at-risk families. Journal of Specialists in Pediatric Nursing. 2001;6(2):73-82. doi:10.1111/j.1744-6155.2001.tb00124.x

24. MacMillan HL, Wathen CN, Barlow J, Fergusson DM, Leventhal JM, Taussig HN. Interventions to prevent child maltreatment and associated impairment. Lancet. 2009;373:250-266. doi:10.1111/j.1744-6155.2001.tb00124.x

25. Arnold DS, O'Leary SG, Wolff LS, Acker MM. The parenting scale: a measure of dysfunctional parenting in discipline situations. Psychol Assess. 1993;5(2):137-144.

26. Rhoades KA, O'Leary SG. Factor structure and validity of the parenting scale. J Clin Child Adolesc Psychol. 2007;36(2):137146. doi:10.1037//1040-3590.5.2.137

27. Prinz RJ, Sanders MR, Shapiro CJ, Whitaker DJ, Lutzker JR. Population-based prevention of child maltreatment: the US Triple P system population trial. Prev Sci. 2009;10(1):1-12. doi:10.1080/15374410701274157

28. World Health Organization (WHO). Preventing child maltreatment: a guide to taking action and generating evidence 2006. http:// apps.who.int/iris/bitstream/10665/43499/1/9241594365_eng. pdf.

29. Turner KM, Richards M, Sanders MR. Randomised clinical trial of a group parent education programme for Australian Indigenous families. J Paediatr Child Health. 2007;43:429-437. doi:10.1046/ j.1440-1754.2002.00077.x-i1

30. López SC, George LM, Herrera ALD, et al. Parenting and physical punishment: primary care interventions in Latin America. Revista Panamericana de Salud Pública. 2000;8(4):257-267. doi:10.1590/s1020-49892000000900005

31. El-Mohandes AA, Katz KS, El-Khorazaty MN, et al. The effect of a parenting education program on the use of preventive pediatric healthcare services among low-income, minority mothers: a randomized, controlled study. Pediatrics. 2003;111(6):13241332. doi:10.1542/peds.111.6.1324

32. Fujiwara T, Kato N, Sanders MR. Effectiveness of group positive parenting program (Triple $\mathrm{P}$ ) in changing child behavior, parenting style, and parental adjustment: an intervention study in Japan. J Child Fam Stud. 2011;20:804-813. doi:10.1007/ s10826-011-9448-1

33. Gylfason G, Sigurðardóttir Z, Skúlason S, et al. Private parenting coach : an intervention that improves the parenting techniques of parents with a long history of social service support. Sálfræðiritið. 2009;14:93-101.

34. Kirby JN, Matthew RS. A randomized controlled trial evaluating a parenting program designed specifically for grandparents. Behav Res Ther. 2014;52:35-44. doi:10.1016/j.brat.2013.11.002 